\title{
Elevated Plasma Corticotrophin Release Factor Levels and In Utero Meconium Passage
}

\author{
JAYARAMAN LAKSHMANAN, SURESHBABU N. AHANYA, VIRENDER REHAN, NOBORU OYACHI, AND MICHAEL G. ROSS
}

Department of Obstetrics and Gynecology, Harbor-UCLA Medical Center, Torrance, California, 90502

\begin{abstract}
Intrauterine meconium (MEC) passage and aspiration may result in significant newborn morbidity, though there is little understanding of the physiologic mechanisms for MEC passage. We hypothesized that stress induces fetal MEC passage via corticotrophin releasing factor (CRF), a known mediator of colonic motility in adult rats. Pregnant rats at e22 were subjected to acute hypoxia or normoxia for $35 \mathrm{~min}$, after which rats were anesthetized and fetuses operatively delivered. Amniotic fluid bilirubin and intestinal alkaline phosphatase were measured as markers for MEC passage, and fetal and maternal plasma CRF and corticosterone levels determined. Hypoxic stress induced defecation in all dams and provoked visible MEC passage in all fetuses. Amniotic fluid bilirubin content was significantly higher in hypoxic fetuses versus controls (1.064 \pm 0.101 versus $0.103 \pm 0.003$ O.D. at $410 \mathrm{~nm}$ ) and intestinal alkaline phosphatase was consistently elevated in MEC stained amniotic fluid. Hypoxia significantly increased plasma CRF (maternal, $82 \pm 5$ to $196 \pm 14 \mathrm{pg} / \mathrm{mL}$; fetal, $284 \pm 15$ to $1523 \pm 185 \mathrm{pg} / \mathrm{mL})$ and corticosterone (maternal, $417 \pm 50$ to $1150 \pm 50 \mathrm{ng} / \mathrm{mL}$; fetal, $96 \pm$ 5 to $182 \pm 10 \mathrm{ng} / \mathrm{mL}$ ) compared with controls. In view of the known action of CRF in adult colonic motility, these results suggest that hypoxic stress-mediated MEC passage in term fetal rats is mediated by a CRF dependent pathway. (Pediatr Res 61: 176-179, 2007)
\end{abstract}

$\mathrm{N}^{\mathrm{e}}$ ewborn MEC passage, a developmentally programmed event, normally occurs within the first 24-48 h after birth, though little is known of the regulatory processes. More than half a million infants born annually in United States are reported to pass MEC in utero. Five to twelve percent of infants with amniotic fluid MEC aspirate MEC during intrauterine life or labor, contributing to MEC aspiration syndrome (1) with accompanying respiratory distress and pulmonary hypertension. In addition to the neonatal effects, in utero, MEC can stimulate umbilical cord vessel constriction, vascular necrosis, and thrombi and lead to ischemic cerebral palsy [see (2) for review]. Four percent of infants with in utero MEC die from associated sequelae, accounting for $2 \%$ of all perinatal deaths (3). Despite the significant morbidity and mortality, little is known of the cascade of events that lead to MEC passage in the newborn following birth or to the mechanisms contributing to "premature" MEC passage in utero.

Received July 31, 2006; accepted September 13, 2006

Correspondence: Michael G. Ross, M.D., Department of Obstetrics and Gynecology, Harbor-UCLA Medical Center, 1000 W. Carson St., Box 3, Torrance, CA 90502; e-mail: mikeross@ucla.edu

Presented at the 2006 Annual Meeting of the Pediatric Academic Society, San Francisco, CA, April 29-May 2, 2006

Supported by a grant from the March of Dimes.

DOI: $10.1203 / p d r .0 b 013 e 31802 d 8 a 81$
In utero MEC passage rarely occurs before 32 wk of gestation and most babies with MEC stained amniotic fluid are 37 wk or older $(1,4)$. The incidence of MEC stained amniotic fluid increases with the gestational age, rarely occurring in preterm infants (4) though reaching as high as $30 \%$ in postterm pregnancies $(5,6)$. Both gastrointestinal maturation and stress have been attributed as risk factors for fetal in utero MEC passage. Evidence in support of "fetal stress" as the cause for the in utero MEC passage comes from frequent clinical observation of MEC passage in association with hypoxic stress $(4,7)$. An increased incidence of MEC passage into amniotic fluid in association with perinatal infection provides further support that fetomaternal stress factors, independent of fetal maturation, may trigger in utero MEC passage (8). However, at present there is no small animal model to support whether in utero MEC passage is mediated either by maturation and/or stress dependent pathways.

CRF, a 41 amino acid polypeptide, was the first identified member of the stress hormone family (9). CRF was originally isolated from ovine hypothalamus (10) and later established to be an important regulator of hypothalamo-pituitary-adrenal axis in a number of species (11-13). CRF is also expressed in abundant amounts in human placenta with increased amounts at term (14), and is positively regulated by glucocorticoids $(15,16)$.

In adult animals and humans, stress is known to increase colonic motility and defecation $(17,18)$, though only recently have the role of stress hormones and stress hormone receptors in stress-mediated defecation been elucidated (19-21). In adult rats, both peripheral and central administration of CRF has been shown to induce defecation during time of stress $(20,22)$. The motility and defecation provoked by peripheral and central CRF mimic those induced by acute stress (23-25).

In the present study, we tested the hypothesis that stressinduced in utero MEC passage in the fetus is analogous to stress-induced defecation in adult rats, and likely mediated by CRF pathway. We tested our hypothesis using an acute hypoxic stress paradigm in term fetal rats.

\section{MATERIALS AND METHODS}

Animals. Time-dated Sprague-Dawley pregnant rats of $14 \mathrm{~d}$ gestation (term $=22 \mathrm{~d}$; Harlan, San Diego, CA) were housed individually and maintained on a standard diet and water ad libitum in a controlled environment (0600-1800 h lights on). A total of 24 pregnant rats were used in this

Abbreviations: CRF, corticotrophin releasing factor; MEC, meconium 
study. Approval for the study was obtained from the Institutional Animal Care and Review Committee.

Experimental technique. For hypoxic stress induction, pregnant rats on d 22 of gestation were placed in a Plexiglas chamber $(18$ " $\times 10$ " $\times 10$ ") fitted with a flow-through system and perfused with a gas mixture of $3 \% \mathrm{O}_{2}, 5 \%$ $\mathrm{CO}_{2}$ and balanced nitrogen. Oxygen flow was adjusted in a stepwise fashion to decrease the oxygen content from $21 \%$ to $16 \%$ to $12 \%$ to $8 \%$. Rats were maintained for 5 min each at $16 \%$ and $12 \%$ oxygen, after which they were exposed to $8 \%$ oxygen for $25 \mathrm{~min}$ before sacrifice. Changes in oxygen content were instituted over a 2 -min period. The oxygen concentration of the chamber was monitored through an outlet fitted with an oxygen analyzer (Nellcor, Mallinckrodt, Hazelwood, MO). Control rats were exposed to $21 \%$ oxygen in an identical chamber for a similar duration. At the end of experimental period, fecal pellets expelled by hypoxic and normoxic pregnant rats were counted, after which rats were rapidly anesthetized by exposure to isoflurane. The abdomen was quickly opened, and the uterine horns with fetuses exteriorized and examined for in utero MEC passage as visible through the uterus.

Individual fetal sacs were carefully removed, membranes surrounding the fetus opened, and amniotic fluids collected using a 1-mL plastic syringe. The fluids were transferred to Microfuge tubes and placed on ice during collection. The tubes were later centrifuged at 13,000 rpm for $20 \mathrm{~min}$ to obtain cell-free amniotic fluid. Fetal heads were decapitated and trunk blood collected using heparinized borosilicate capillary tubes (CardinalHealth, Dublin, OH). The capillary tubes were transferred to plastic tubes and centrifuged to obtain plasma, which was stored at $-80^{\circ} \mathrm{C}$ in presence of aprotinin until hormone assays performed. Placental tissues were harvested at sacrifice, snap frozen in liquid nitrogen, and later processed for CRF quantification. Following the removal of the uterine horn with fetuses, maternal blood was collected via cardiac puncture and placed in centrifuge tubes containing EDTA and aprotinin. Samples were centrifuged and plasma separated for hormonal quantification. At the end of the study, maternal rats were euthanized with excess isoflurane and disposed.

MEC quantification. MEC passage was quantified by the amniotic fluid bilirubin content determined by spectrophotometric absorption at $410 \mathrm{~nm}$ (26) and expressed as OD per milliliter of amniotic fluid. In addition, pooled amniotic fluids were examined for the presence of intestinal alkaline phosphatase, as a marker for the bulk movement of intestinal content, by Western blot analysis as detailed below.

Western blotting. Briefly, aliquots of pooled amniotic fluid collected from control and hypoxic fetuses were mixed with saline (1:3). An aliquot was mixed with equal volumes of $2 \times$ Laemmli's electrophoretic sample buffer, heated in a boiling water bath for $5 \mathrm{~min}$, cooled and centrifuged. The supernatants (35 $\mu \mathrm{L}$ per well) were subjected to SDS-PAGE and proteins resolved on a $7.5 \%$ separating gel. Prestained Bio-Rad molecular weight markers were co-run with samples. Proteins were transferred to Immun-blot PVDF membrane (Bio-Rad, Hercules, CA) and immunoblotted with a rabbit polyclonal antiserum (1:2000) to bovine intestinal alkaline phosphatase (Sigma Chemical Co., St. Louis, MO). Immunoreactive bands identified by chemiluminescence using SuperSignal West Pico Chemiluminescent substrate (Pierce, Rockford, IL).

$\boldsymbol{C R F - R I A}$. Plasma and placental CRF concentrations were quantified using CRF-RIA kit (Peninsula Laboratories, San Carlos, CA). Briefly, CRF from plasma $(0.5-1.5 \mathrm{~mL})$ was measured using the protocol described by the manufacturer except that two Sep-Pak C18 cartridges (Waters Associated, Marlboro, MA) connected in tandem were used for CRF extraction, as described by Emanuel et al. (27). The Sep-Pak columns were eluted by $60 \%$ acetonitrile in $1 \%$ trifluoroacetic acid and extracts were then dried under Savant speed- vacuum concentrator. The dried materials were reconstituted with $0.5 \mathrm{~mL}$ RIA buffer, centrifuged in a Microfuge at $12,000 \mathrm{rpm}$ for $30 \mathrm{~min}$ and stored frozen at $-20^{\circ} \mathrm{C}$. CRF from placenta was measured following acetic acid extraction procedure (28). Briefly, individual placental tissues (400-500 mg wet weight) were placed in glass test tubes containing $1 \mathrm{~mL}$ of acetic acid $(1 \mathrm{M})$, heated in a boiling water bath for $10 \mathrm{~min}$, and cooled to room temperature. Four milliliters of methanol (50\% in $0.5 \mathrm{M}$ acetic acid) were added to each tube and placental extracts homogenized using UltraTurrex tissue homogenizer. The homogenates were centrifuged at $15,000 \mathrm{~g}$ for $30 \mathrm{~min}$ and supernatants transferred to plastic tubes and dried under speed-vacuum concentrator. The pellet was reconstituted in $1.0 \mathrm{~mL}$ RIA buffer, centrifuged, and supernatant used for RIA. CRF concentrations were measured in duplicate at two different dilutions. The assay sensitivity was 6 $\mathrm{pg} /$ tube, with intra- and interassay coefficients of variation of $9 \%$ and $12 \%$, respectively. Recovery studies performed with unlabelled CRF ranged between $80 \%$ and $85 \%$ both for plasma and placental extracts. Plasma CRF levels were expressed as picograms per milliliter plasma while placental CRF contents expressed nanogram per milligram wet weight. Blood obtained from six hypoxia and six control maternal rats bearing similar number of fetuses was used for plasma CRF analysis. Blood from all fetuses of a litter was pooled and treated as one sample. Six samples for each group were used for analysis. Three placenta removed from each animal $(n=18$ placentas in each group) were processed for CRF analysis.

Data analysis. All values are expressed as mean \pm SEM. Comparisons between normoxic and hypoxic treatment groups were made using unpaired $t$ test. A $p$ value $<0.05$ was considered as significant.

\section{RESULTS}

Compared with control pregnant rats $(n=12)$ exposed to $21 \%$ oxygen, hypoxic pregnant rats $(n=12)$ exhibited copious defecation when the oxygen content of the chamber reached $12 \%$. The number of fecal pellets expelled by hypoxic pregnant rats was significantly higher than normoxic pregnant rats $(9 \pm 1$ versus $5 \pm 1, p<0.001)$. Hypoxia significantly increased plasma CRF $(82 \pm 5$ to $196 \pm 14 \mathrm{pg} / \mathrm{mL}, p<0.001$; Fig. $1 A)$ and corticosterone (417 \pm 50 to $1150 \pm 50 \mathrm{ng} / \mathrm{mL}$, $p<0.001$; Fig. $1 B$ ) concentrations in hypoxic pregnant rats compared with control dams.

Upon opening the uterine wall, all hypoxic and control fetuses were alive. The number of fetuses varied between 8 and 12 per litter. In hypoxic fetuses, the MEC passage was consistently evident as yellowish coloration visibly discernable when examined through the uterine wall (Fig. 2). Yellow discoloration of skin and placenta and presence of intestinal particulate material adhering to the placenta or the fetal body were common in the hypoxic fetuses. No similar MEC passage was observed in the normoxic group. Bilirubin content
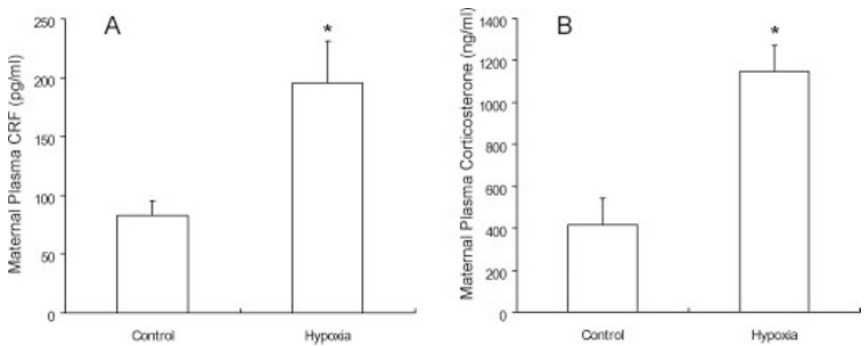

Figure 1. Maternal plasma $\operatorname{CRF}(A)$ and corticosterone $(B)$ levels following hypoxic and normoxic periods. ${ }^{*} p<0.001 v s$ normoxic.

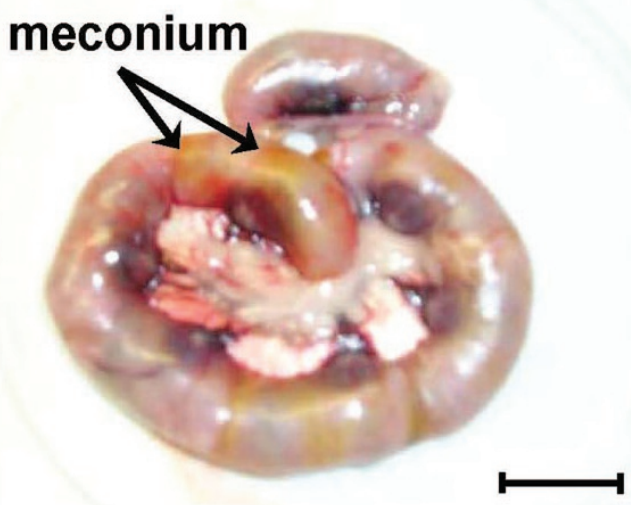

Figure 2. Photograph $(3 \times)$ showing fetal meconium staining at the end of hypoxia exposure as visualized through the fetal sac. Note the presence of yellowish meconium staining throughout the uterine horn. Scale bar represents $1 \mathrm{~cm}$. 

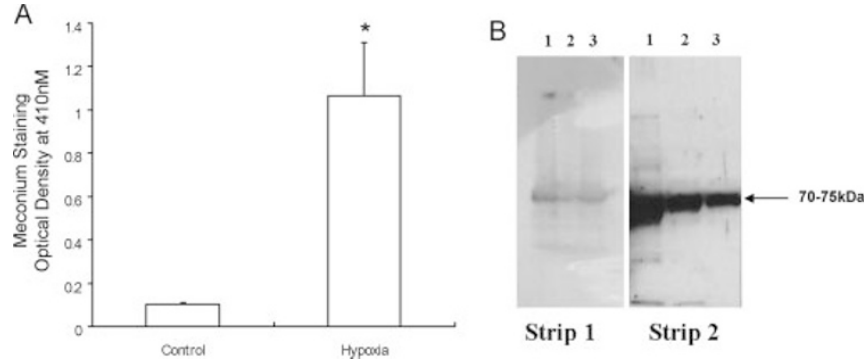

Figure 3. Analysis of amniotic fluids for meconium staining. (A) Amniotic fluid OD $(410 \mathrm{~nm})$ of individual hypoxic and normoxic fetuses. Each column represents the mean \pm SEM of 24 fetuses. $* p<0.001 v s$ normoxic. (B) Amniotic fluid intestinal alkaline phosphatase immunoreactive bands as visualized by Western blot analysis (representative result). Each number represents pooled amniotic fluid from each litter. Note the faint $70-75 \mathrm{kD}$ alkaline phosphatase band in the amniotic fluids of normoxic fetuses (Strip 1) and the strong $70-75 \mathrm{kD}$ alkaline immunoreactive band in amniotic fluids of hypoxic fetuses (Strip 2).
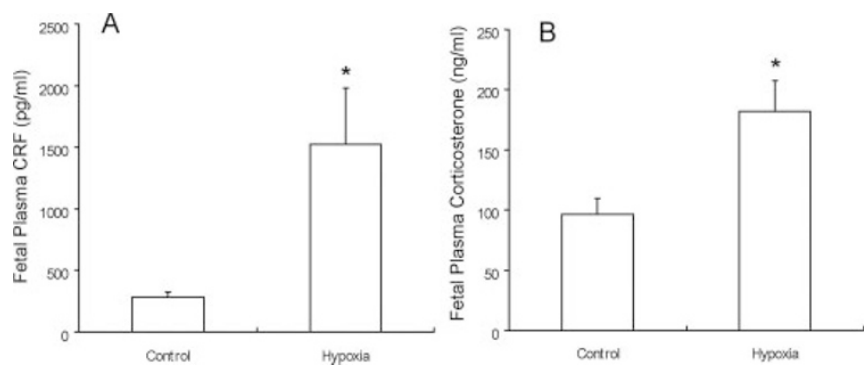

Figure 4. Fetal plasma CRF $(A)$ and corticosterone $(B)$ levels following hypoxic and normoxic periods. ${ }^{*} p<0.001 v s$ normoxic.

was significantly elevated in amniotic fluid collected from hypoxic compared with control fetuses (OD at $410 \mathrm{~nm}: 1.064$ \pm 0.101 versus $0.103 \pm 0.003, p<0.001$; Fig. $3 A$ ). Western blot analysis confirmed a marked increase in intestinal alkaline phosphatase in the amniotic fluid of fetuses subject to hypoxic treatment (Fig. 3B). Hypoxia significantly increased fetal plasma CRF (284 \pm 15 to $1523 \pm 185 \mathrm{pg} / \mathrm{mL}, p<0.001$; Fig. $4 A$ ) and corticosterone (96 \pm 5 to $182 \pm 10 \mathrm{ng} / \mathrm{mL}, p<0.001$; Fig. $4 B$ ) concentrations compared with control fetuses.

Placental CRF levels were not significantly different between hypoxic and control animals $(0.765 \pm 0.073$ versus $0.845 \pm 0.067 \mathrm{ng} / \mathrm{mg}$ wet weight, $p=\mathrm{NS})$.

\section{DISCUSSION}

The present study was designed to test the hypothesis that fetal in utero MEC passage is analogous to stress-mediated defecation in adult rats in its regulation by CRF. We chose acute hypoxia as a stress paradigm since MEC staining is frequently observed in human newborns in association with hypoxic stress $(4,7)$. Exposure of pregnant rats (as detailed above) to a gradually decreasing oxygen concentration, in a time-dependent manner, consistently stimulated fetal in utero MEC passage on d 22 of gestation. Copious defecation in pregnant rats was also observed under the hypoxic condition.

A number of studies in adult rats indicate that stress is a potent stimulator of increased colonic motility and defecation $(17,18)$. Interestingly, peripheral administration of CRF has been reported to induce a characteristic pattern of cecocolonic myoelectric activity, activation (i.e. c-fos expression) of myenteric neurons and stimulation of colonic transit and defecation $(20,29)$. Exposure of isolated colonic segments to CRF and CRF antagonists has been shown to elicit opposite effects on both mechanical and electrical peristaltic activity, suggesting that endogenous CRF may have direct effects on colonic motor function (30). Studies with the specific CRF-R1 antagonist indicate that CRF-mediated cecocolonic myoelectric activity, myenteric neuron activation, and defecation primarily use the CRF-R1 system (20). Recently, evidence for CRF-R1 localization at the level of colonic myenteric nervous system in adult rat colon has been documented (31).

In the present study, significant increases in plasma CRF levels were observed in pregnant rats subjected to hypoxia compared with control pregnant rats exposed to normoxia. Increases in plasma CRF levels in pregnant rats suggest that hypoxic stress-mediated defecation in these rats is triggered, at least in part, by peripheral CRF pathways. However, further studies are required to determine whether the observed defecation in pregnant rats is mediated via colonic CRF-R1 as demonstrated in adult rats with restraint stress (20).

MEC passage in fetal rats was consistently observed after pregnant rats were exposed to the hypoxia paradigm. All fetuses were alive at the end of the study and responded in a similar fashion irrespective of their position in uterine horn. Increased intestinal alkaline phosphatase content and bilirubin concentrations in amniotic fluid of all fetuses exposed to hypoxia confirmed MEC passage. Plasma CRF levels were significantly elevated in MEC stained fetuses, suggesting that fetal MEC passage also may be modulated by CRF.

In the present study, we did not identify the specific source(s) of plasma CRF. Pregnancy is the only time at which high levels of plasma CRF are evident, and the human placenta has been identified to be the major source of maternal and fetal plasma CRF $(32,33)$. Similarly, it is likely that plasma CRF in both pregnant rats and their fetuses originates from the placenta (32). In the present study, despite the marked increase in plasma CRF levels of both fetuses and pregnant rats, there was no decrease in placental CRF content in response to hypoxia. As the increases in fetal and maternal plasma CRF content represents only a small percentage of individual and pooled placental CRF content, respectively, these changes would not detectable by RIA techniques. It is also possible that the release of CRF stems from sources other than placenta. Studies in adult mice report the existence of specific unidirectional brain-to-blood transport system for CRF (34). Furthermore, this transport system is acutely modulated by adrenal steroids suggesting the participation in the control of stress response (35). In the present investigation, we have observed marked increases in plasma corticosterone levels both in pregnant rats and fetuses under hypoxic stress condition suggesting the possibility that plasma glucocorticoids may have stimulated transport of brain CRF to plasma in hypoxic groups.

In summary, in utero MEC passage observed in the present study is evidence of a stress-associated event in term fetal rats. Although the origin of plasma CRF is not known, elevated plasma CRF is the likely mediator of hypoxic stress stimulated 
defecation in pregnant rats and in utero MEC passage in fetuses. This rat model should be useful to delineate the molecular mechanisms underlying the in utero MEC passage and possibly to develop an antenatal therapy to prevent the development of MEC aspiration syndrome.

Acknowledgments. The authors thank Elena Bronshtein, Ron Beloosesky, M.D., Ashley Berry, Amanda Berry, and Reuben Lakshmanan for their participation in hypoxic studies. We also thank Glenda Calvario for performing plasma and placental hormone assays.

\section{REFERENCES}

1. Cleary GM, Wiswell TE 1998 Meconium-stained amniotic fluid and the meconium aspiration syndrome. An update. Pediatr Clin North Am 45:511-529

2. Ahanya SN, Lakshmanan J, Morgan BL, Ross MG 2005 Meconium passage in utero: mechanisms, consequences, and management. Obstet Gynecol Surv 60:45-56

3. Wiswell TE, Tuggle JM, Turner BS 1990 Meconium aspiration syndrome: have we made a difference? [see comments]. Pediatrics 85:715-721

4. Wiswell TE 2001 Handling the meconium-stained infant. Semin Neonatol 6:225-231

5. Miller FC, Read JA 1981 Intrapartum assessment of the postdate fetus. Am J Obstet Gynecol 141:516-520

6. Usher RH, Boyd ME, McLean FH, Kramer MS 1988 Assessment of fetal risk in postdate pregnancies. Am J Obstet Gynecol 158:259-264

7. Richey SD, Ramin SM, Bawdon RE, Roberts SW, Dax J, Roberts J, Gilstrap LC 1995 Markers of acute and chronic asphyxia in infants with meconium-stained amniotic fluid. Am J Obstet Gynecol 172:1212-1215

8. Katz VL, Bowes WA Jr 1992 Meconium aspiration syndrome: reflections on a murky subject. Am J Obstet Gynecol 166:171-183

9. Perrin MH, Vale WW 1999 Corticotropin releasing factor receptors and their ligand family. Ann N Y Acad Sci 885:312-328

10. Vale W, Spiess J, Rivier C, Rivier J 1981 Characterization of a 41-residue ovine hypothalamic peptide that stimulates secretion of corticotropin and beta-endorphin. Science 213:1394-1397

11. Rivier CL, Plotsky PM 1986 Mediation by corticotropin releasing factor (CRF) of adenohypophysial hormone secretion. Annu Rev Physiol 48:475-494

12. Challis JR, Brooks AN 1989 Maturation and activation of hypothalamic-pituitary adrenal function in fetal sheep. Endocr Rev 10:182-204

13. Lovejoy DA, Balment RJ 1999 Evolution and physiology of the corticotropinreleasing factor (CRF) family of neuropeptides in vertebrates. Gen Comp Endocrinol 115:1-22

14. Frim DM, Emanuel RL, Robinson BG, Smas CM, Adler GK, Majzoub JA 1988 Characterization and gestational regulation of corticotropin-releasing hormone messenger RNA in human placenta. J Clin Invest 82:287-292

15. Robinson BG, Emanuel RL, Frim DM, Majzoub JA 1988 Glucocorticoid stimulates expression of corticotropin-releasing hormone gene in human placenta. Proc Natl Acad Sci U S A 85:5244-5248
16. Jones SA, Brooks AN, Challis JR 1989 Steroids modulate corticotropin-releasing hormone production in human fetal membranes and placenta. J Clin Endocrinol Metab 68:825-830

17. Narducci F, Snape WJ, Battle WM, London RL, Cohen S 1985 Increased colonic motility during exposure to a stressful situation. Dig Dis Sci 30:40-44

18. Hall CS 1934 Emotional behavior in the rat. I. Defecation and urination as measures of individual differences in emotionality. J Comp Psychol 18:385-403

19. Williams CL, Peterson JM, Villar RG, Burks TF 1987 Corticotropin-releasing factor directly mediates colonic responses to stress. Am J Physiol 253:G582-G586

20. Maillot C, Million M, Wei JY, Gauthier A, Tache Y 2000 Peripheral corticotropinreleasing factor and stress-stimulated colonic motor activity involve type 1 receptor in rats. Gastroenterology 119:1569-1579

21. Martinez V, Tache Y 2001 Role of CRF receptor 1 in central CRF-induced stimulation of colonic propulsion in rats. Brain Res 893:29-35

22. Monnikes H, Raybould HE, Schmidt B, Tache Y 1993 CRF in the paraventricular nucleus of the hypothalamus stimulates colonic motor activity in fasted rats. Peptides 14:743-747

23. Bueno L, Collins SM, Junien JL 1989 Stress and Digestive Motility. John Libby Publishers, London

24. Rubio CA, Sveander M, Duvander A 1989 A model to evaluate acute and chronic stress in the colonic mucosa of rats. Dis Colon Rectum 32:26-29

25. Castagliuolo I, Lamont JT, Qiu B, Fleming SM, Bhaskar KR, Nikulasson ST, Kornetsky C, Pothoulakis C 1996 Acute stress causes mucin release from rat colon: role of corticotropin releasing factor and mast cells. Am J Physiol 271:G884-G892

26. Molcho J, Leiberman JR, Hagay Z, Hagay Y 1986 Spectrophotometric determination of meconium concentration in amniotic fluid. J Biomed Eng 8:162-165

27. Emanuel RL, Robinson BG, Seely EW, Graves SW, Kohane I, Saltzman D, Barbieri R, Majzoub JA 1994 Corticotrophin releasing hormone levels in human plasma and amniotic fluid during gestation. Clin Endocrinol (Oxf) 40:257-262

28. Takahashi K, Totsune K, Murakami O, Saruta M, Nakabayashi M, Suzuki T, Sasano H, Shibahara S 2004 Expression of urocortin III/stresscopin in human heart and kidney. J Clin Endocrinol Metab 89:1897-1903

29. Bonaz B, Tache Y 1994 Water-avoidance stress-induced c-fos expression in the rat brain and stimulation of fecal output: role of corticotropin-releasing factor. Brain Res 641:21-28

30. Mancinelli R, Azzena GB, Diana M, Forgione A, Fratta W 1998 In vitro excitatory actions of corticotropin-releasing factor on rat colonic motility. J Auton Pharmacol 18:319-324

31. Chatzaki E, Crowe PD, Wang L, Million M, Tache Y, Grigoriadis DE 2004 CRF receptor type 1 and 2 expression and anatomical distribution in the rat colon. J Neurochem 90:309-316

32. Goland RS, Wardlaw SL, Stark RI, Brown LS Jr, Frantz, AG 1986 High levels of corticotropin-releasing hormone immunoactivity in maternal and fetal plasma during pregnancy. J Clin Endocrinol Metab 63:1199-1203

33. Goland RS, Wardlaw SL, Blum M, Tropper PJ, Stark RI 1988 Biologically active corticotropin-releasing hormone in maternal and fetal plasma during pregnancy. Am J Obstet Gynecol 159:884-890

34. Martins JM, Kastin AJ, Banks WA 1996 Unidirectional specific and modulated brain to blood transport of corticotropin-releasing hormone. Neuroendocrinology 63:338348

35. Martins JM, Banks WA, Kastin AJ 1997 Acute modulation of active carriermediated brain-to-blood transport of corticotropin-releasing hormone. Am J Physiol 272(2 pt 2):E312-E319 